УДК $537.86 ; 621.37$

\title{
ВРАЩЕНИЕ ПЛОСКОСТИ ПОЛЯРИЗАЦИИ ДВУСЛОЙНЫМИ ПЛОСКО-КИРАЛЬНЫМИ СТРУКТУРАМИ. ОБЗОР РЕЗУЛЬТАТОВ ТЕОРЕТИЧЕСКИХ И ЭКСПЕРИМЕНТАЛЬНЫХ ИССЛЕДОВАНИЙ
}

\author{
А. А. КИРИЛЕНКО ${ }^{1,3}$, С. А. СТЕШЕНКО ${ }^{1,3}$, В. Н. ДЕРКАЧ ${ }^{1}$, С. А. ПРИКОЛОТИН ${ }^{1}$, Д. Ю. КУЛИК ${ }^{1}$, \\ С. Л. ПРОСВИРНИН ${ }^{2,3}$, Л. П. МОСБПАН ${ }^{1}$ \\ ${ }^{1}$ Институт радиофизики и электроники Национальной Академии наук Украины, \\ Украина, Харьков, 61085, ул. Проскуры 12 \\ ${ }^{2}$ Институт радиоастрономии Национальной Академии наук Украины,, \\ Украина, Харьков, 61002, ул. Краснознаменная, 4 \\ ${ }^{3}$ Харьковский национальный университет, \\ Украина, Харьков, 61077, пл. Свободы, 4
}

\begin{abstract}
Аннотация. Представлены примеры, иллюстрирующие поиск различных двухслойных метаматериалов, обеспечивающих вращение плоскости поляризации («оптическая активность»). Подбор объектов иллюстрирует двадцатилетнюю историю поиска нового принципа создания вращателей поляризации на планарных метаматериалах, реализованных в виде тонкослойных периодических решеток. Само проявление оптической активности как таковой, наличие или отсутствие удовлетворительного или идеального согласования, возможность многополосного эффекта, роль высших пространственных гармоник в «электродинамике» явления объясняются спецификой собственных колебаний возбуждаемых в зазоре многослойной структуры.
\end{abstract}

Ключевые слова: 2D киральность; двуслойный экран; двуслойная диафрагма; оптическая активность; диэдральная симметрия; собственные колебания

\section{1. ВВЕДЕНИЕ}

Первые публикации о так называемых метаматериалах, нашедших неожиданные применения в микроволновой технике и оптике, вызвали всплеск исследований. В первую очередь они были сосредоточены на новых полезных свойствах 3D объектов со сложной геометрией, ранее слабо изученных. Это стало возможным благодаря быстрому прогрессу в электродинамическом моделировании и открывшимся возможностям в свободном манипулировании 3D формой объектов.

Далее остановимся только на одном из новых электродинамических эффектов, наблюдаемых в метаматериалах - вращении плоскости поляризации волны, прошедшей через плоско-киральную композитную решетку или соответствующую волноводную диафрагму"

* Предварительные материалы данной статьи доложены на конференции MSMW2016 (Харьков, 2016): A. Kirilenko, S. Prosvirnin, A. Perov, S. Steshenko, V. Derkach, N. Kolmakova, S. Prikolotin, D. Kulik, "On the nature and general regularities of optical activity in planar-chiral double-layer metamaterials," (Invited paper), Proc. of the 9 Int. Kharkiv Symp. on Physics and Engineering of Microwaves, Millimeter and Submillimeter Waves (MSMW'2016), Kharkiv, Ukraine, June 21-24, 2016, 6 p. 


\section{БИБЛИОГРАФИЧЕСКИЙ СПИСОК}

1. Holloway, C. L.; Kuester, E. F.; Gordon, J. A.; O'Hara, J.; Booth, J.; Smith, D. R. An overview of the theory and applications of metasurfaces: the two-dimensional equivalents of metamaterials. IEEE Antennas and Propagation Magazine, vol. 54, No. 2, p. 10-35, 2012. DOI : 10.1109/MAP.2012.6230714.

2. Pendry, J. B. A chiral route to negative refraction. Science, vol. 306, No. 5700, 2004, p.1353-1355. DOI: 10.1126/science. 1104467 .

3. Arnaut, L. R.; Davis, L. E. On planar chiral structures. Progress in Electromagnetic Research Symposium (PIERS 1995), 24-28 July, Seattle, WA, p. 165.

4. Arnaut, L. R. Chirality in multi-dimensional space with application to electromagnetic characterisation of multi-dimensional chiral and semi-chiral media. Journal of Electromagnetic Waves and Applications, vol. 11, No. 11, p. 1459-1482, $1997 . \quad$ DOI: 10.1163/156939397X00549.

5. Prosvirnin, S. L. Analysis of electromagnetic wave scattering by plane periodical array of chiral strip elements. Proc. of 7th Int. Conf. on Complex Media «Bianisotropics-98», 3-6 June 1998, 185-188, Technische Universitat Braunschweig, Germany. DOI: 10.13140/2.1.1744.1929.

6. Prosvirnin, S. L. Transformation of polarization when waves are reflected by a microstrip array made of complex-shaped elements. Journal of Communications Technology and Electronics, vol. 44, No. 6, p. 635-639, 1999.

7. Kwon, D.-H.; Werner, P. L.; Werner, D. H. Optical planar chiral metamaterial designs for strong circular dichroism and polarization rotation. Optics Express, vol. 16, No. 16, p. 11802-11807, 2008. DOI: 10.1364/OE.16.011802.

8. Decker, M.; Ruther, M.; Kriegler, C. E.; Zhou, J.; Soukoulis, C. M.; Linden, S.; Wegener, M. Strong optical activity from twisted-cross photonic metamaterials. Optics Letters, vol. 34, No 16, p. 2501-2503, 2009. DOI: 10.1364/OL.34.002501.

9. Rogacheva, A. V.; Fedotov, V. A.; Schwanecke, A. S.; Zheludev, N. I. Giant gyrotropy due to electromagnetic-field coupling in a bilayered chiral structure. Phys. Rev. Lett., vol. 97, p. 177401, 2006. DOI: 10.1103/PhysRevLett.97.177401.

10. Mackay, A. Proof of polarization independence and nonexistence of crosspolar terms for targets presenting $n$-fold $(\mathrm{n}>2)$ rotational symmetry with special reference to frequency-selective surfaces. Electron. Lett., vol. 25, No 24, p. 1624-1625, 1989. DOI: 10.1049/el:19891088.

11. Sonsilphong, A.; Gutruf, P.; Withayachumnankul, W.; Abbott, D.; Bhaskaran, M.; Sriram, S.; Wongkasem, N. Flexible bi-layer terahertz chiral metamaterials. Journal of Optics, vol. 17, No. 8, p. 085101, 2015. DOI: 10.1088/2040-8978/17/8/085101.

12. Plum, E.; Zhou, J.; Dong, J.; Fedotov, V. A.; Koschny, T.; Soukoulis, C. M.; Zheludev, N. I. Metamaterial with negative index due to chirality. Phys. Rev. B, vol. 79, p. $035407, \quad 2009$. DOI: 10.1103/PhysRevB.79.035407.

13. Zarifi, D.; Soleimani, M.; Nayyeri, V. Dual- and multiband chiral metamaterial structures with strong optical activity and negative refraction index. IEEE Antennas Wireless Propag. Lett., vol. 11, p. 334-337, 2012. DOI: 10.1109/LAWP.2012.2191261.

14. Zarifi, D.; Soleimani, M.; Nayyeri, V.; Rashed-Mohassel, J. On the miniaturization of semiplanar chiral metamaterial structures. IEEE Trans. Antennas Propag., vol. 60, No. 12, p. 5768-5776, 2012. DOI: 10.1109/TAP.2012.2214015.

15. Zarifi, D.; Soleimani, M.; Nayyeri, V. A novel dual-band chiral metamaterial structure with giant optical activity and negative refractive index. J. Electromagn. Waves Appl., vol. 26, p. 251-263, 2012. DOI: 10.1163/156939312800030767.

16. Gordon, R.; Brolo, A. G.; McKinnon, A.; Rajora, A.; Leathem, B.; Kavanagh, K. L. Strong polarization in the optical transmission through elliptical nanohole arrays. Phys. Rev. Lett., vol. 92, No. 3, p. 037401, 2004. DOI: 10.1103/PhysRevLett.92.037401.

17. Derkach, V.; Kirilenko, A.; Salogub, A.; Prikolotin, S.; Kolmakova, N.; Ostrizhnyi, Ye. Giant optical activity in artificial planar-chiral sructures. Proc. of Int. Kharkov Symp. MSMW'13, 23-28 Jun. 2013, Kharkov, Ukraine. IEEE, 2013, p. 435-438. DOI: 10.1109/MSMW.2013.6622098.

18. Li, Z.; Zhao, R.; Koschny, T.; Kafesaki, M.; Alici, Kamil Boratay; Colak, E.; Caglayan, H.; Ozbay, E.; Soukoulis, C. M. Chiral metamaterials with negative 
refractive index based on four ' $U$ ' split ring resonators. Appl. Phys. Lett., vol. 97, p. 081901, 2010. DOI: 10.1063/1.3457448

19. Kirilenko, A.; Kolmakova, N.; Prikolotin, S. Plane-chiral pair with opposite rotatios as a new way to rotate polarization up to $90^{\circ}$. Proc. of Int. Conf. MMET, 28-30 Aug. 2012, Kharkiv, Ukraine. IEEE, 2012, p. 80-83. DOI: 10.1109/MMET.2012.6331155.

20. Kolmakova, N.; Prikolotin, S.; Perov, A.; Derkach, V.; Kirilenko, A. Polarization plane rotation by arbitrary angle using D4 symmetrical structures. IEEE Trans. Microwave Theory Tech., vol. 64, No. 2, p. 429-435, 2016. DOI: 10.1109/TMTT.2015.2509966.

21. Li, Z.; Caglayan, H.; Colak, E.; Zhou, J.; Soukoulis, Costas M.; Ozbay, E. Coupling effect between two adjacent chiral structure layers. Optics Express, vol. 18, No. 6, p. 5375-5383, 2010. DOI: 10.1364/OE.18.005375.

22. Maslovski, S. I.; Morits, D. K.; Tretyakov, S. A. Symmetry and reciprocity constraints on diffraction by gratings of quasi-planar particles. J. Opt. A, Pure Appl. Opt., vol. 11, No. 7, p. 074004, 2009. DOI: 10.1088/1464-4258/11/7/074004.

23. Колмакова, Н. Г.; Кириленко, А. А.; Просвирнин, С.Л. Плоско-киральные диафрагмы в круглом волноводе и проявления «оптической активности». Радиофизика и радиоастрономия, Т. 16, № 1, p. 70-81, 2011.

URL: http://journal.rian.kharkov.ua/index.php/ra/article/view/4 54.

24. Кириленко, А. А.; Сенкевич, С. Л.; Тысик, Б. Г. Закономерности резонансных явлений в открытых структурах волноводного типа. Радиотехника $u$ электроника, Т. 35, № 4, p. 687-694, 1990.

25. Cornwell, J. F. Appendix C: Character tables for the crystallographic point groups. In Group Theory in Physics: An Introduction. New York, NY, USA: Academic, 1997.

26. Kolmakova, N.; Prikolotin, S.; Kirilenko, A.; Perov, A. Simple example of polarization plane rotation by the fringing fields interaction. Proc. of European Microwave Conf., 6-10 Oct. 2013, Nuremberg. IEEE,
2013, p. 936-938. URL: http://ieeexplore.ieee.org/document/6686812/.

27. Кириленко, А. А.; Колмакова (Дон), Н. Г.; Приколотин, С. А. Сверхкомпактная 90 скрутка на основе пары плоско-киральных диафрагм в квадратном волноводе. Известия вузов. Радиоэлектроника, Т. 55, № 4, C. 31-35, 2012. URL: http://radio.kpi.ua/article/view/S002134701204005X.

28. Kirilenko, A. A.; Perov, A. O. On the common nature of the enhanced and resonance transmission through the periodical set of holes. IEEE Trans. Antennas Propag., vol. 56, No. 10, p. 3210-3216, 2008. DOI: 10.1109/TAP.2008.929437.

29. Колмакова (Дон), Н.Г.; Перов, А. О.; Сенкевич, С. Л.; Кириленко, А. А. Аномальное прохождение ЭМВ сквозь запредельные отверстия и собственные колебания волноводных объектов и периодических структур. Известия вузов. Радиоэлектроника, Т. 54, № 3, p. 3-13, 2011. URL: http://radio.kpi.ua/article/view/S0021347011030010.

30. Kirilenko, A. A.; Tysik, B. G. Connection of S-matrix of waveguide and periodical structures with complex frequency spectrum. Electromagnetics, vol. 13, No. 3, p. 301-318, 1993. DOI: 10.1080/02726349308908352.

31. Munk, B. A. Frequency Selective Surfaces: Theory and Design. New York: Wiley, 2000.

32. Perov, A. O.; Kirilenko, A. A.; Derkach, V. N. Polarization response manipulation for compound circular hole fishnet metamaterial. IEEE Antennas Wireless Propag. Lett., vol. 16, p. 117-120, April 2016. DOI: 10.1109/LAWP.2016.2559452.

33. Кулик, Д. Ю.; Перов, А. О.; Мосьпан, Л.П.; Колмакова, Н.Г. Компактные вращатели плоскости поляризации на основе диафрагм с прямоугольными щелями. Радиофизика и электроника, т. 20, № 2, c. $96-101,2015$.

34. Кулик, Д.Ю.; Стешенко, С.А.; Кириленко, А.А. Компактные вращатели плоскости поляризации на заданный угол в квадратном волноводе. Радиофизика и электроника, т. 22, № 1, С. 15-20, 2017.

Поступила в редакцию ? По-сле переработки question 